\title{
APROXIMACIÓN AL ESTUDIO DE LAS COLOCACIONES EN ÁRABE MARROQUí
}

\author{
Houda JAAFAR*
}

Universidad Sidi Mohammed Ben Abdellah

BIBLID [1133-8571] 26 (2019) 13.1-11.

Resumen: El árabe marroquí mantiene una relación muy fuerte con la cultura del pueblo, ya que es el verdadero vehículo de los valores y de las experiencias humanas. En este sentido, nos interesa estudiar una parte muy importante de la fraseología del árabe marroquí: las colocaciones. Intentaremos responder en esta investigación a estas preguntas: ¿Qué es una colocación?, ¿Existe una diferencia entre expresión fija y colocación? ¿Cómo se reparten las colocaciones en árabe marroquí?

Palabras clave: Árabe marroquí, Colocación, Cultura, Glosario.

Abstract: «Approximation to the study of collocations in Moroccan Arabic». Moroccan Arabic has a very strong relationship with the culture of the people because it is the true vehicle of human values and experiences. In this regard, we are interested in studying a very important part of the phraseology of Moroccan Arabic: collocations. We will try to answer the following questions in this research: What is a collocation? Is there a difference between set expressions and collocations? How are the collocations distributed in Moroccan Arabic?

Key words: Moroccan Arabic, Collocation, Culture, Glossary.

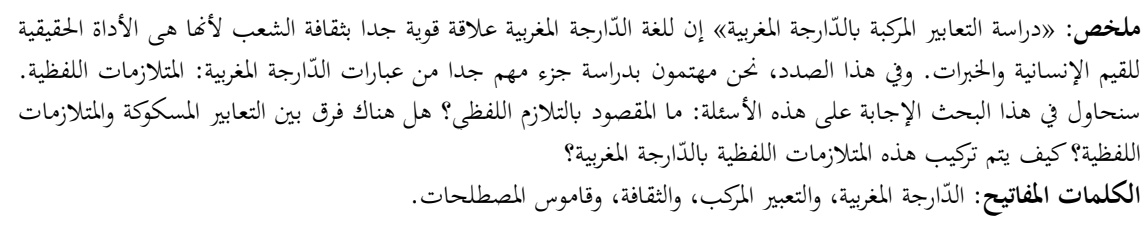

* Estudiante en segundo año de doctorado. E-mail: houdajaafar1992@gmail.com 


\section{Introducción}

El árabe marroquí está formado por un conjunto de variedades habladas que se practican en distintas regiones. La influencia del tamazight y de las lenguas europeas (francés y español) sobre el árabe marroquí marca algunas de las diferencias morfológicas, sintácticas, fonéticas y lexicales entre éste y el árabe estándar.

El uso frecuente de un conjunto de palabras unidas como denominaciones o expresiones por parte de una comunidad determinada o un grupo preciso, ha favorecido el estudio de la fraseología como una disciplina de la lingǘstica. Hoy en día, el estudio fraseológico es muy imprescindible, ya que permite enriquecer nuestro conocimiento sobre aspectos asociados con la semántica, la sintaxis y la estilística. Según el Diccionario Básico de Lingüística (2005: 100), la fraseología es el «conjunto de frases hechas, locuciones figuradas, metáforas y comparaciones fijadas, modismos y refranes existentes en una lengua».

La importancia de la fraseología es cada vez mayor y atrae el interés de muchos investigadores, tanto en el marco teórico como el marco práctico, sobre todo, en el aspecto relacionado con la composición de diccionarios y el estudio de las lenguas extranjeras.

Antes de pasar a nuestro objeto de estudio, la colocación en el árabe marroquí ${ }^{1}$, debemos conocer cómo se clasifican las colocaciones en español. Para ello presentamos la clasificación ofrecida por Corpas (1996):

- Sustantivo (sujeto) + verbo: en este tipo de colocación, el verbo marca una característica del sustantivo (sea persona o cosa).

- Verbo + sustantivo (objeto): estas colocaciones se caracterizan por la pertenencia del colocado y colocativo al mismo campo semántico.

- Adjetivo + sustantivo: los adjetivos, en estas colocaciones, desempeñan el papel del colocativo, además pertenecen a una terminología determinada.

- Sustantivo + preposición+ sustantivo: en estas colocaciones, el segundo sustantivo es la base o el núcleo de la colocación, mientras que el primer sustantivo es el colocativo.

- Verbo + adverbio: los adverbios, en estas colocaciones, son de dos tipos: adverbios de modo y adverbios de intensidad.

(1) Para las colocaciones en árabe clásico, cf. Santillán 2019. 
- Adjetivo + adverbio: sobre esta, Corpas (1996: 74) dice «aquí incluimos colocaciones de participio en función adjetival y adverbio».

Nuestro interés por el estudio de la colocación en el árabe marroquí es reciente. En este artículo, nuestro objetivo es estudiar qué se entiende por colocación, si existe una diferencia entre expresión fija y colocación y cómo se reparten las colocaciones en árabe marroquí.

\section{La colocación en árabe marroquí}

El término «colocación» apareció por primera vez en la lingüística inglesa y su marco teórico fue definido por Firth (1951). Las colocaciones son expresiones que consisten en dos palabras (un colocado y un colocativo). El colocado es el núcleo de la colocación que no se puede ni sustituir ni modificar. El colocativo, al contrario, es una palabra que admite la modificación o la sustitución. Además, cada constituyente de la colocación guarda su sentido literal. Ejemplos:

$$
\begin{aligned}
& \text { يدير لمزيان } \quad y d \bar{r} \text { l-mazyān «hacer el bien» }
\end{aligned}
$$

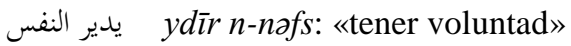

$$
\begin{aligned}
& \text { يدير الطايلة ydìr t-tâyyla «construir su futuro» }
\end{aligned}
$$

En estos tres ejemplos, el verbo $d \bar{a} r-y d \bar{r} r$ (hacer) se combina de forma casi ilimitada con otras palabras.

Así, se puede decir que la colocación es una construcción en donde el núcleo determina la elección de las otras palabras. Además, se advierte que hay una autonomía de los constituyentes ya que se puede substituir el colocativo como hemos mencionado en la definición.

\subsection{La distinción entre la expresión fija y la colocación}

Para distinguir entre expresión fija y colocación, nos basamos sobre los criterios dados por Liang (1991-1992). Según este autor, la collocation se démarque de l'expression figée sur trois plans:

- L'autonomie des composants,

- Leur inaltérabilité sémantique 
- Et la substitution possible du collocatif (Mekhilef 2005: 19). ${ }^{2}$

Es decir, en una colocación cada componente guarda su valor semántico, no existe un sentido metafórico en la colocación y se puede sustituir un componente. Mientras que en una expresión fija, los componentes se caracterizan por su sentido polisémico y por la relación mantenida entre sí.

\subsection{Tipos de la colocación en árabe marroquí}

Durante nuestra investigación y recopilación del corpus de los ejemplos ilustrativos, al analizar las colocaciones, no hemos encontrado más de dos tipos de colocaciones: las nominales y las verbales.

\subsubsection{Colocación nominal}

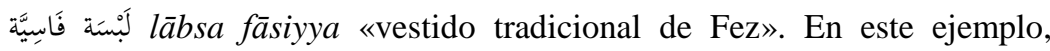
hablamos solo de lābsa (vestido tradicional) pero añadiendo fāsiyya (de Fez) llegamos a un nivel más alto desde el punto de vista expresivo. La colocación en su totalidad se refiere a un vestido que llevan las novias en sus bodas que es típico de Fez.

El vestido tradicional no es siempre $f a \bar{s} i$, ya que hay vestidos de otras ciudades; como el vestido de Rabat, lābsa rbātiyya, el vestido de Salé, lābsa slāwiya, etc. Aquí, se advierte el contenido semántico. Y esta característica relaciona el vestido tradicional con la ciudad por razones de costura ya que cada ciudad tiene una forma típica de costura y de modelos. Sin embargo, la elección de este adjetivo, fāsiyya, podría deberse a otra razón: se trata de una expresión denominativa, disponible en la lengua y que se refiere a un estereotipo sobre el vestido tradicional de Fez.

\subsubsection{Colocación verbal}

La colocación verbal es una construcción que se articula alrededor de un verbo núcleo. El verbo tạh يط يطيح

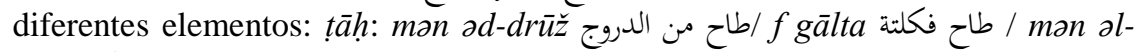
fūq طاح من الفوق. Que corresponden respectivamente a: «caer: de las escaleras / en

(2) «Según Liang, la colocación se diferencia de la expresión fija en tres niveles: la autonomía de sus componentes, su inalterabilidad semántica y la posible sustitución del colocativo» (traducción propia). 
un charco / de lo alto». La facultad de generar tales construcciones es ilimitada: un $\mathrm{V}$ (el núcleo) + CC de lugar.

Hay otros verbos que tienen un valor polisémico como $x d a-y \bar{a} x u d$

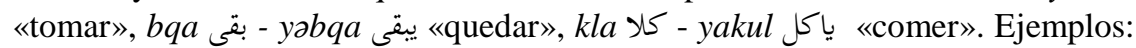

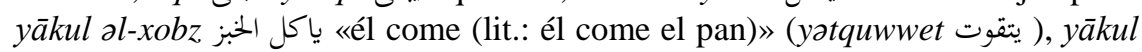

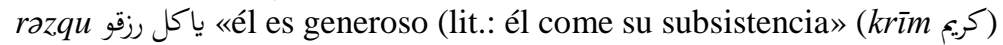

La colocación verbal se define por tres factores:

a) La equivalencia: toda la colocación verbal equivale a un verbo extraído del

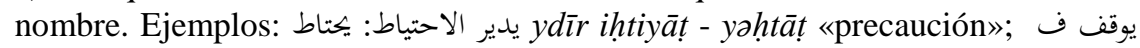
ydīr al-holl - yhall «solucionar».

b) La permutación: la construcción puede cambiar a pasiva, pero la forma activa sigue siendo la más utilizada. Ejemplo: تلف له الراي Ł tlaf lu r-rāy $\rightarrow$ rāyu tālaf «perdido».

c) La conmutación: el verbo puede conmutarse para describir el progreso en un

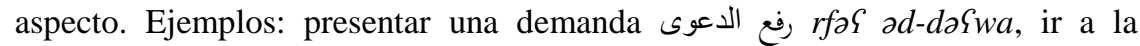

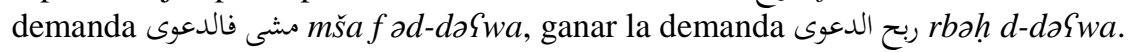

\section{Taxonomía de las colocaciones}

\subsection{Las colocaciones nominales}

El núcleo de las colocaciones nominales es un sustantivo. Mientras, el colocativo puede ser otro sustantivo o bien un adjetivo.

\subsubsection{Sustantivo + sustantivo}

a) Ejemplos ilustrativos

\begin{tabular}{|c|c|c|}
\hline Traducción & Transcripción & Las colocaciones \\
\hline Chica de una familia modesta & bənt Swām & ل بَنْتْ عْوَام \\
\hline Demonio necrófago & $b \bar{u}-x \partial n \check{s} a$ & بُو خَنْشَة \\
\hline Taller de bordado & dār mSollma & دَارْ المْعَلْمَة \\
\hline Año de hambruna & Sām al-būn & عَاْمُ الْبُونْ \\
\hline Chica guapa & lalla mama & لالة ماما \\
\hline
\end{tabular}


b) El significado de la colocación

- bənt گwām. Swām: «grupo de individuos que pertenece a una misma sociedad, cultura o una patria común, habla la misma lengua y vive en el mismo territorio». bənt $\{w \bar{a} m$ : en la antigüedad, había en Marruecos chicas destinadas solamente a servir en las casas de los jueces, «cadi». En la cultura marroquí, esta colocación denota una chica cuya familia es modesta, ordinaria.

- bū-xənša. $b u$ : viene del árabe estándar, significa «el padre de». Se usa en la cultura marroquí para designar el padre de alguien o de algo, como la raíz. $x ə n s ̌ a: ~ « b o l s a ~ o ~ s a c o »$, aquí se refiere a la bolsa de harina. En cuanto a su fabricación, está hecha de varias formas, siendo el material de lana o de plástico. bu-xənša: es un vagabundo, conocido por este apodo porque siempre va llevando una bolsa de harina en su espalda, la cual es reutilizada para poner cualquier cosa, sin pensar en su peso. Nadie conoce su nombre. En la cultura marroquí, las mujeres lo utilizan para dar miedo a sus hijos para que dejen de hacer locuras infantiles o bien para que duerman.

- dār al-mৎallma. dār «casa». mSallma «dueña, diseñadora, maestra de artesanía». dār al-mSallma «taller de bordado». El bordado es considerado como una de las artesanías que se han asociado con la vida de las mujeres, tales como coser y tejer. Se practica más dentro de los hogares de forma permanente o estacional y además de los modelos hechos a mano, existe la máquina que dio la posibilidad de diversificar los productos. Las chicas bordan ropa de las mujeres de varios tipos, ropa de cama, de mesas, etc. dār al-mSəllma era una casa antigua ubicada en las principales ciudades, especialmente Fez. Las madres iban a esta casa con sus hijas y las dejaban allí un período de tiempo para que aprendieran costura, bordado y todo tipo de trabajos manuales. También, para que las niñas fueran capaces de asumir la responsabilidad del hogar, incluso las chicas mimadas en las casas de sus padres y las chicas que estudiaban iban a trabajar allí. Desde el punto de vista de las madres, la chica debía conocer y practicar una artesanía en paralelo con sus estudios.

- $\{\bar{a} m$ əl-būn. $९ \bar{a} m$ «año». əl-būn: «el bono», fórmula escrita que consiste en el derecho de una persona a requerir prestación. $€ \bar{a} m$ al-būn «año de hambruna». ৎăm tā̌̌ y $९ \bar{a} m$ al-būn: se trata de dos denominaciones distintas, pero tienen un mismo significado, ya que la propagación de epidemias y enfermedades 
contagiosas están relacionadas con la falta de higiene, hambruna, etc. La utilización de estas palabras es por lo tanto la consecuencia de la interacción de todos los factores mencionados. De hecho, la expresión ৎām al-būn está vinculada a un contexto socio-histórico bien definido, se acuñó como resultado de las necesidades alimenticias y la pobreza que Marruecos experimentó en los años 1939 y 1940. La palabra būn es un préstamo del francés bon, que significa, en árabe marroquí, «bono, vale, cupón, recibo».

- lalla mama. lalla «señora». La palabra mama es un apodo que califica a las chicas por su belleza. Se utiliza mucho en algunas ciudades de Marruecos, como Rabat y Fez. Las chicas y las mujeres marroquíes vivían en unidades familiares, en «familias cerradas» o en un harén, donde las familias extendidas vivían como una sola unidad, y donde las mujeres estaban aisladas y requerían del permiso de los hombres para salir de la vivienda. lalla mama tiene un papel importante a nivel cultural, ya que forma parte del patrimonio marroquí. Ha sido el título de una canción muy famosa cuyo autor es anónimo.

\subsubsection{Sustantivo + adjetivo}

a) Ejemplos ilustrativos

\begin{tabular}{|c|c|c|}
\hline Traducción & Transcripción & Las colocaciones \\
\hline Tela de muselina & tūb hayāti & توب حَيَاتي \\
\hline Alheña de las primeras damas & hənna mazwārāt & حَنَّةْْْ مَزْوَراتِتْ \\
\hline Cementerio que superó cien años. & rawḍa mansiyya & رَوْْضَة مَنْسِيَّة \\
\hline Taza de té bien preparada & $k \bar{a} s$ mšahḩ̣r & كَاسن مْشَحََّرٌ \\
\hline Jabón negro & șābūn boldi & صَابُونْ بَلْدِي \\
\hline Pintalabios & ¿karfasi & عْكَْ فَاسِى \\
\hline
\end{tabular}

b) El significado de la colocación

- tūb hayāti. tūb «tela», hayāti «muselina, variedad de tela». tūb hayāti «tela hecha de algodón». Antes, las mujeres marroquíes cosían con esta tela pantalones y todo tipo de servilletas; también la utilizaban en la cocina. Las mujeres marroquíes de hoy en día la usan mucho para hacer pañuelos.

- hənna məzwārāt. ḩønna «alheña», planta que crece en climas cálidos y secos. El tatuaje de alheña es temporal. La manera más extendida de aplicarla es 
hacerlo sobre la piel. Hay distintos dibujos, entre los marroquíes encontramos, por ejemplo, el saharaui, el $x ə t f a$, el $f a ̄ s i$, etc. La práctica de la alheña fascina a nuestra sociedad, es una parte de nuestras costumbres y tradiciones. También, forma parte de las ceremonias de boda, de las ceremonias de imposición del nombre a los recién nacidos, e incluso es una marca de hospitalidad para los huéspedes que vienen de lejos (entre las mujeres). La práctica del tatuaje de la alheña está presente en otras culturas tales como la India, la China y en distintos países africanos. məzwārāt: forma plural de məzwāra «mujer cuya unión como pareja ha durado mucho tiempo y cuya relación con su esposo es muy fuerte». Es la mujer que pone alheña a la novia en su cuerpo cuando se baña en la casa de sus padres. En la cultura popular es un buen presagio para la chica que se casa por primera vez.

- rawda mansiyya: rawda «cementerio». mansiyya es el participio pasivo del verbo nsa - yənsa, «olvidar». rawda mansiyya «cementerio abandonado, olvidado», donde ya no se entierra más. Nadie se atreve a tocar este tipo de cementerios debido a su carácter sagrado por los cuerpos que en él hay enterrados. Esta denominación está justificada por la naturaleza del terreno cementerio, que es como una tierra que no es cultivable.

- kās mšaḥhor. kās «vaso ». mšahḥor «bien preparado». kās mšaḥhor «taza de té bien preparada». Con el participio mš $h$ ḥor nos referimos al té que tiene un color rojo oscuro. àtāy, como lo llaman los marroquíes. Tiene una gran importancia en la gastronomía marroquí, hasta tener sus rituales y hábitos. Incluso, apareció entre los artesanos una nueva artesanía dedicada a la fabricación de herramientas para preparar el té: bandejas, jarras de té, rbāyə̧ (unos utensilios donde se pone el azúcar), etc. El té forma parte de la vida del pueblo marroquí. Las reuniones (fiestas, invitaciones entre familias, y otros.) han impuesto la presencia permanente del té. Está presente en el patrimonio literario marroquí, por ejemplos, hay muchos poetas que hablan del té en sus casidas.

- șābūn baldi. șābūn «jabón». baldi «local, del país». Es de color negro y se conoce simplemente como baldi. El șābūn baldi, «jabón negro» o «jabón beldi» es una pasta negra hecha de aceite de oliva, se caracteriza por sus propiedades exfoliantes naturales. Es el producto esencial en un ḩəmmām marroquí. 
- 'kar fāsi «pintalabios hecho con amapolas». Es un cosmético totalmente natural, en polvo, extraído de pétalos de amapola y cáscaras de granada secas. Las mujeres lo usan como pintalabios, también lo utilizan con otros ingredientes para el cuidado de sus cuerpos y cabellos.

\subsection{Las colocaciones verbales}

Como su denominación lo indica, estas colocaciones tienen como núcleo un verbo. El colocativo puede cambiar de una categoría a otra, como verbo + nombre, verbo + preposición + nombre u otro tipo:

a) Ejemplos ilustrativos

\begin{tabular}{|c|c|c|}
\hline Traducción & Transcripción & Las colocaciones \\
\hline sé un hombre & kūn rāžal & كُونْ رَجْل \\
\hline ponte en buena posición & $q \partial d d$ al-waqfa & قَادٌٌ الْوقْفَة \\
\hline siéntete cómodo & $x \bar{u} d$ rāhtok & حُود رَاحْتَكُُْ \\
\hline
\end{tabular}

b) El significado de las colocaciones

- kūn rāžəl. kūn: imperativo del verbo kān - ykūn «ser estar», está en segunda persona del singular. $r a \bar{z} z l$ «hombre». kūn rāžal «jsé hombre!». Cuando alguien deja un asunto entre las manos de otro, le dice esto, es decir, que sea responsable, no tenga miedo de nada y que se esté orgulloso de él. También se emplea cuando uno quiere casarse, entonces su padre se lo dice con el sentido de ser un hombre capaz de construir una familia.

- qadd al-waqfa. qadd «ponte», imperativo en segunda persona del singular. $ə l$ waqfa «la posición». qadd al-waqfa, expresión que se dice cuando alguien está mal colocado o situado.

- $x \bar{u} d$ rāhtık. $x \bar{u} d$, imperativo del verbo $x d a$ - yāxud «coger, tomar». rahtək «tu descanso, tu libertad». $x \bar{u} d$ rəh̆tək: esta expresión se dice como forma de cortesía cuando viene un huésped a visitar alguien. También significa tomar el tiempo necesario para hacer algo, o bien sentirse cómodo a la hora de hablar de un asunto. 


\section{Conclusión}

Los estudios lingüísticos consultados, ponen el acento en el aspecto social y cultural de estas expresiones, que son recursos abundantes en la memoria colectiva del pueblo.

En árabe marroquí, las colocaciones se dividen en dos tipos: nominales y verbales. El núcleo es la parte fija de una colocación, no sufre ninguna modificación. Mientras, el colocativo se caracteriza por la posibilidad de ser modificado o sustituido por otro colocativo que puede ser de otra categoría gramatical. Además, las colocaciones en el árabe marroquí no tienen la misma clasificación española. En español, se pueden encontrar colocaciones adjetivales que en árabe marroquí se agrupan bajo la misma taxonomía de las colocaciones nominales.

Hemos querido con este artículo dar a conocer parte del patrimonio lingüístico del árabe marroquí a través de las expresiones expuestas. Para recopilar estos ejemplos, se han tenido en cuenta dos pasos. El primero, agrupar las colocaciones en árabe marroquí y el segundo buscar su equivalente en español. De esta forma se ha facilitado la comprensión de los mismos al lector y se ha puesto de manifiesto la correspondencia lingǘstica entre las dos lenguas. Aún así, queremos ser modesto y reconocer que hemos ofrecido al lector «como muestra, un botón».

Finalmente, quisiéramos indicar que los ejemplos aportados en este trabajo son solamente el punto de partida con el que hemos iniciado un trabajo de investigación sobre las colocaciones en árabe marroquí que arrojará más datos en un futuro.

\section{BIBLIOGRAFÍA}

ABBOUD-HAGGAR, Soha. 2010². Introducción a la dialectología de la lengua árabe. Granada, Fundación El Legado Andalusí.

AGUADÉ, Jorge. 2008. «Árabe marroquí (Casablanca)». En: Manual de dialectología neoárabe. En: Estudios Árabes e Islámicos. Estudios de Dialectología Árabe 1. Federico Corriente y Ángeles Vicente, eds. Zaragoza, Instituto de Estudios Islámicos y del Oriente Próximo, pp. 281-310. 
ALVARADO ORTEGA, M. Belén. 2007. «Las formulas rutinarias como unidades fraseológicas». ELUA. Estudios de Lingüística 21, pp. 21-33.

CORPAS PASTOR, Gloria (1996): Manual de fraseología española. Madrid, Gredos.

MEKHILEF, Msaouda. 2005-2006. Etude contrastive d'expressions figées du français et de l'arabe dialectal algérien dans une perspective d'enseignement. Setif, Université Ferhat Abbas.

MOSCOSO GARCÍA, Francisco. 2015. Diccionario de árabe marroquí. En: Bibliotheca Arabo-Romanica et Islamica 10. Gijón, Trea.

LACHKAR, Abdenbi. 2014. Proverbes et locutions stéréotypées du Maroc: Lexicalisation, modalisation, transparence et figement. Paris, Geuthner.

LUNA TRAILL, Elizabeth, VIGUERAS ÁVILA, Alejandra \& ESTELA BAEZ PINAL, Gloria. 2005. Diccionario básico de lingüística. México, Universidad Nacional Autónoma de México.

SANTILLÁN GRIMM, Paula. 2019. "Las colocaciones en árabe: estado de la cuestión". MEAH 68 (2019), pp. 325 -360.

SHABAN MOHAMMAD SALEM, Tarek. 2014. La fraseología en español y en árabe: estudio, comparación, traducción y propuesta de un diccionario. Madrid, Universidad Complutense de Madrid. 